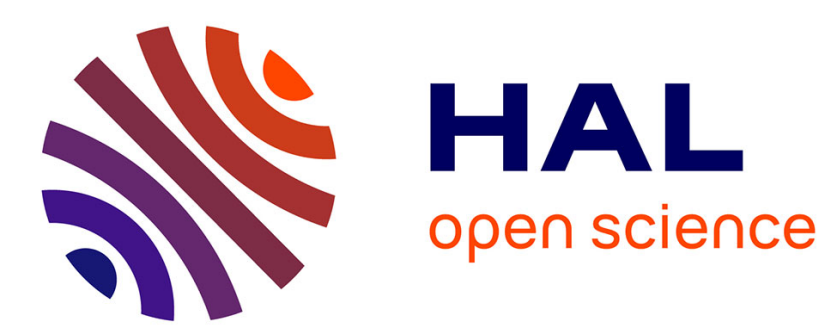

\title{
Modified algorithms for nonconforming taylor discretization
}

\author{
Eytan Kochavi, Reuven Segev, Yosef Yomdin
}

\section{To cite this version:}

Eytan Kochavi, Reuven Segev, Yosef Yomdin. Modified algorithms for nonconforming taylor discretization. Computers \& Structures, 1993, 49 (6), pp.969-979. hal-01068072

\section{HAL Id: hal-01068072 \\ https://hal.science/hal-01068072}

Submitted on 24 Sep 2014

HAL is a multi-disciplinary open access archive for the deposit and dissemination of scientific research documents, whether they are published or not. The documents may come from teaching and research institutions in France or abroad, or from public or private research centers.
L'archive ouverte pluridisciplinaire HAL, est destinée au dépôt et à la diffusion de documents scientifiques de niveau recherche, publiés ou non, émanant des établissements d'enseignement et de recherche français ou étrangers, des laboratoires publics ou privés. 


\title{
MODIFIED ALGORITHMS FOR NONCONFORMING TAYLOR DISCRETIZATION
}

\author{
E. Kochavi, $†$ R. SEgEV † and Y. Yomdin $\ddagger$ \\ †The Pearlstone Center for Aeronautical Engineering Studies, Department of Mechanical Engineering, \\ Ben-Gurion University of the Negev, P.O. Box 653, Beer-Sheva 84105, Israel \\ ‡Department of Theoretical Mathematics, The Weizmann Institute of Science, Rehovot 76100, Israel
}

\begin{abstract}
Algorithms for solving partial differential equations which extend previous applications of the nonconforming Taylor discretization method (NTDM) are presented. In one modification the number of interrelated grid points is variable, thus enabling additional geometric flexibility. Another modification is the approximation of the governing differential equation using the method of weighted residuals. A simple one-dimensional test case with a known analytic solution is solved using this code. The results demonstrate that precision is enhanced when using the method of weighted residuals with an increased number of interrelated points. The algorithm is applied as a general purpose two-dimensional code for nonlinear steady state heat-conduction. Two-dimensional examples with complex geometry and boundary conditions are then solved both by the NTDM and by the finite elements method (FEM). The results obtained by the two methods are compared.
\end{abstract}

\section{INTRODUCTION}

In a previous article [1] an application of the nonconforming Taylor discretization method (NTDM) for the solution of a partial differential equation (PDE), was introduced. This method is based on a general scheme proposed by Yomdin and Elihai [2] for manipulating approximations of $k$-times differentiable functions as an application of Kolmogorov's results [3] about the optimal way to memorize a $k$-times differentiable function up to accuracy $\epsilon$. Yomdin and Elihai suggested that the approximation be represented by the coefficients of $k$-jets ( $k$ th-order Taylor expansions of the function) at a collection of preselected nodes. The scheme does not require that the expansions about distinct nodes coincide anywhere, and there is a discrepancy between the values that the distinct jets assume at any point in the domain (see Fig. 1). By the properties of the Taylor approximations, for a given $\epsilon>0$ there is an $h=O\left(\epsilon^{1 /(k+1)}\right)$ such that the error in the approximation and its derivatives is smaller than $\epsilon$. Hence, for the case where the function is approximated by jets about the selected nodes, if the distance between two nodes is small enough the discrepancy of the values and the values of the derivatives may be kept smaller than $\epsilon$.

The nonconforming Taylor discretization method uses this approach for the approximate representation of a function in order to approximate an unknown solution of a differential equation. It follows that the various unknown jets have to satisfy some 'compatibility' conditions in addition to the conditions offered by the differential equation itself. Such conditions will imply that the jets correspond to local approximations of a smooth function. Following the guidelines of the general scheme, it is natural to require that the discrepancy will be of the same order of magnitude as the error resulting from the Taylor approximation. Thus, we do not spend unnecessary computational effort on fitting the local expansions with a discrepancy smaller than the error we expect for the given grid size and order of expansion.

In [1] the NTDM was applied to an irregular grid with a fixed number of five neighboring points as a result of the order of jets used. The differential equation was satisfied only locally for each grid-point (collocation method). In the present work, algorithms are introduced which enable the application of the NTDM with two modifications: An algorithm for implementing the PDE by the method of weighted residuals is presented and, using an averaging process, the number of neighboring nodes may be increased. These modifications suggest better geometrical flexibility in the construction of the grid and result in improved accuracy.

Several authors have used approximations of functions by jets in a flexible grid in order to yield the finite difference formulation of given partial differential equations. However, in all cases exact fitting of the local expansions was a basic condition. No general algorithm for solving any PDE has been suggested either. Perrone and Kao [4] used jet approximations and suggested an averaging process to increase the number of neighboring points. They showed that this was useful in avoiding singularity and improving the accuracy. Liszka and Orkisz [5] suggested an efficient averaging process for 


$$
x_{2}-x_{1}<h ; \quad\left|u_{1}(h)-u_{2}(0)\right|<\varepsilon
$$

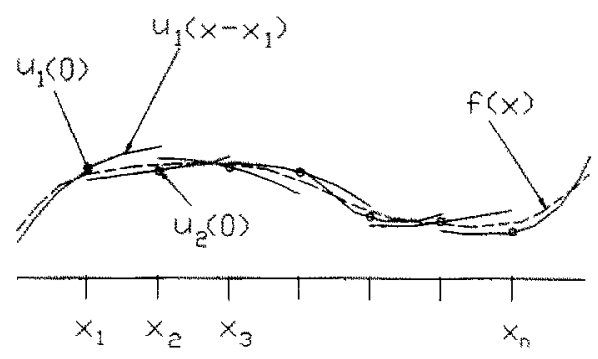

Fig. 1. Schematic drawing of nonconforming jet approximation of a function (shown dashed).

performing the same task. The main advantages of the present applications of the NTDM over those in $[4,5]$ are: (a) the use of the permitted discrepancy to reduce computation effort, and (b) a general formulation that is easily applied to different types of PDEs.

The application of the NTDM to the numerical solution of engineering field problems consists of two independent steps: the controlled discrepancy (continuity, discontinuity) and the implementation of the differential equation. As for the differential equation, its representation is independently developed and implemented. As an example for the application of the method to field problems, two-dimensional nonlinear heat conduction was designated. This should be viewed, however, as a representative example of a wider family of engineering problems such as structural torsion or membrane stretching. An application of the method to two-dimensional elasticity will be given in a forthcoming work [6].

It is evident that there is a great potential in further development of the NTDM. The results of this work indicate that the combination of controlled discrepancy between jets and the estimation of the truncation error, leads to improved efficiency. Additional motivation is provided by the work of Sonnemans et al. [7], who used the Taylor series expansions for the solution of ordinary differential equations, and showed that efficiency can be considerably improved by increasing the order of the Taylor series approximation. In the present work only second jets were used, but the order will be increased in further works. Yomdin and Wiener [8] address some theoretical aspects related to the solution of ordinary differential equations by the jet approach.

\section{FORMULATION OF THE ALGORTTHMS}

In the following sections the formulation of some applications of the NTDM is described in three parts. In the first part the implementation of the PDE in two alternative methods is presented. The second part describes the implementation of the connectivity and boundary condition equations with controlled discrepancy. The third part describes the arrangement and solution of the algebraic equations.

\subsection{The implementation of the PDE}

Consider the operator equation describing a boundary value problem for the function $u$ in the domain $\Omega$

$$
A u=f \text { in } \Omega
$$

where $u$ is required to satisfy some boundary conditions. Among the various variational approximation methods available for finding an approximate solution of this problem, we choose to use the collocation method and the method of weighted residuals. While the application of the collocation method was introduced in [1], we will emphasize here the application of the method of weighted residuals.

Consider the $N$ nodal points distributed arbitrarily in the domain $\Omega$ and some additional nodes distributed on the boundary (see Fig. 2). As mentioned in the introduction the approximate solution will be represented by a collection of $N k$-jets $\left\{u_{1}, i=1, \ldots, N\right\}$ about the internal nodes. With each node $i$, we associate a subdomain $\Omega_{i}$ of $\Omega$ containing a number of nodes on its boundary. We consider the solution of the partial differential equation in the domain $\Omega$, subject to boundary conditions offered by the yet unknown values of the function at the boundary nodes of $\Omega_{i}$. It is possible that some of the neighboring nodes are genuine boundary nodes. (Eventually, as mentioned in the introduction and as will be described in the next section, these conditions will be satisfied only approximately.)

Consider the base functions for polynomials of order $k$

$$
\begin{aligned}
& \phi_{1}=1 ; \quad \phi_{2}=x^{\prime} ; \quad \phi_{3}=y^{\prime} ; \quad \phi_{4}=x^{\prime} y^{\prime} ; \\
& \phi_{5}=x^{\prime 2} ; \quad \phi_{6}=y^{\prime 2} ; \quad \text { etc },
\end{aligned}
$$

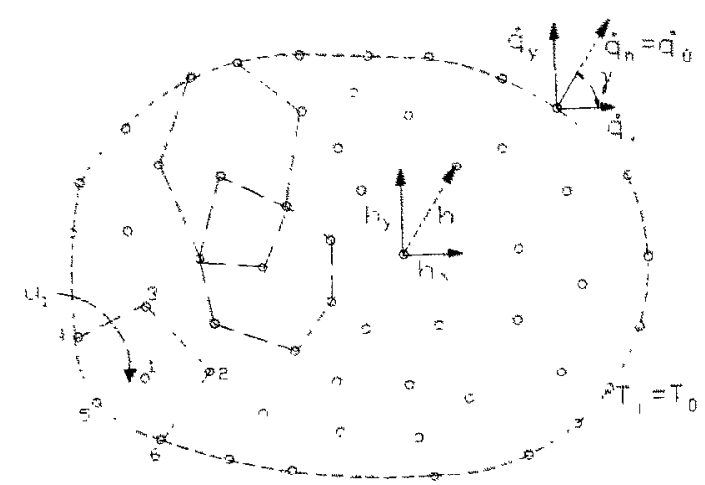

Fig. 2. The discretization of the domain. 
in the local coordinate system: $x^{\prime}=x-x_{i}$; $y^{\prime}=y-y_{i}$. We may write

$$
u_{i}=\sum_{j=1}^{k} c_{i j} \phi_{j} ; \quad i=1, N
$$

where the coefficients $C_{i j}$ are those to be determined in order to solve the local boundary value problem. The substitution of the approximation (2) in the operator equation results in a residual

$$
E\left(x, y, c_{i j}\right) \equiv A\left(u_{i}\right)-f \neq 0 .
$$

The collocation method states that choosing the point $x^{\prime}=y^{\prime}=0$ as the collocation point, the residual at this point is set to zero. Thus, we have to satisfy

$$
\left[A\left(u_{i}\right)-f\right]_{x^{\prime}=y^{\prime}=0}=0 .
$$

The method of weighted residuals obtains the coefficients $c_{i j}$ by setting the integral (over the subdomain $\boldsymbol{\Omega}_{i}$ ) of the weighted residual of the approximation (3) to zero

$$
\int_{\Omega_{i}} v_{i}\left(x^{\prime}, y^{\prime}\right) E\left(x^{\prime}, y^{\prime}, c_{i j}\right) \mathrm{d} x^{\prime} \mathrm{d} y^{\prime}=0,
$$

where $v_{i}$ is the weight function. In principle, additional equations can be written for each subdomain with the use of additional independent weight functions. In the present implementation, however, one weight function for each subdomain will be used so that the implementation of the PDE either by the collocation method or by the weighted residuals method will result in a single equation for the jet's coefficients. Note that we may obtain eqn (4) from the requirement (5) for the problem case where $v_{i}\left(x^{\prime}, y^{\prime}\right)=\delta(0,0)$, the Dirac $\delta$ at the $i$ th node.

To demonstrate a specific implementation of a PDE we consider the example of nonlinear steady state heat conduction. The problem of nonlinear steady state heat conduction is defined by Poisson's equation with boundary conditions of imposed temperature or heat flux. The twodimensional boundary value problem in Cartesian coordinates is

$$
\frac{\partial}{\partial x}\left(k(T) \frac{\partial T}{\partial x}\right)+\frac{\partial}{\partial y}\left(k(T) \frac{\partial T}{\partial y}\right)+\dot{q}=0
$$

Here $T(x, y)$ is the temperature, $\dot{q}(x, y)$ the heat source and we will consider the nonlinear case in which the dependence of the material conductivity $k$ on the temperature is given by

$$
k(T)=k_{s}(1+\alpha T)
$$

The following polynomial function $T_{1}$ is the approximated, or discretized, temperature distribution over this region in the vicinity of node 1

$$
T_{1}(x, y)=a_{1}+b_{1} x^{\prime}+c_{1} y^{\prime}+d_{1} x^{\prime} y^{\prime}+e_{1} x^{\prime 2}+f_{1} y^{\prime 2} .
$$

Throughout this work a polynomial function of order 2 is considered. For the neighborhood of any point $i$, the coefficients $a_{i} \cdots f_{i}$ of the approximation $T_{i}$, are unknown and yet to be determined. In particular, points on the boundary are defined as neighboring points for internal nodes which are located near the boundary, but no polynomial approximation is introduced for boundary points. If we have $N$ internal points, the total number of unknowns will be $N$ times the number of coefficients in the polynomial function, which yields $6 N$ in our case.

To apply the collocation method (4) the jet (8) is substituted into the differential equation (6), differentiating, multiplying by $k(T)$ (with $T$ represented by the polynomial), and finally setting $x_{i}^{\prime}$ and $y_{i}^{\prime}$ to zero (stating that the polynomial function satisfies the differential equation at the central point $i$ ), will result in the equation

$$
2 e+2 f=-\frac{\dot{q}}{k_{s}}-\alpha\left[b^{2}+c^{2}+2 a(e+f)\right]
$$

where $a$ to $f$ are the coefficients which correspond to the central point $i$. In the above equation, the lefthand side contains the linear terms and the righthand side the nonlinear terms, multiplied by $\alpha$. Obviously, in case of temperature independent conductivity ( $\alpha=0$, in eqn (7)), the nonlinearity vanishes also in equation (9).

In order to apply the formulation by the weighted residual method the Poisson equation (6) is substituted in (5)

$$
\int_{\Omega_{i}} v_{i}\left(-\frac{\partial}{\partial x} k(T) \frac{\partial T}{\partial x}-\frac{\partial}{\partial y} k(T) \frac{\partial T}{\partial y}-\dot{q}\right) \mathrm{d} x \mathrm{~d} y=0 .
$$

A weight function $v_{i}$ that is defined to be zero on the boundary of the local subdomain will simplify the form of the weak formulation (10). Using such a weighting function and Green's theorem, the weak formulation takes the form

$$
\int_{\Omega_{i}}\left(\frac{\partial v_{i}}{\partial x} k(T) \frac{\partial T}{\partial x}+\frac{\partial v_{i}}{\partial y} k(T) \frac{\partial T}{\partial y}-v_{i} \dot{q}\right) \mathrm{d} x \mathrm{~d} y=0 .
$$

Here we suggest a function that is $C^{0}$, has a value of one at the local origin and vanishes on the boundary. Figure 3 shows a local discrete domain at node 1 and the local Cartesian coordinates. In addition, $n$ neighboring points define the boundary of the subdomain 


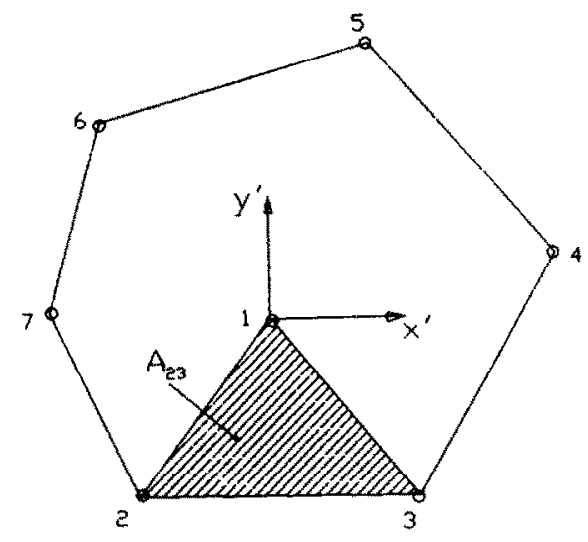

Fig. 3. A local subdomain.

$\Omega_{i}$ (in Fig. 3, $n=6$ for exampie). A weight function that satisfies the above conditions would be an $n$ faced pyramid of height 1 at the local origin node 1 . The function $v$ may be defined, therefore, separately over each triangle (shown hatched in Fig. 3) and for the triangle 123 , it is

$$
v_{23}=\frac{A_{23}-x^{\prime} \Delta y+y^{\prime} \Delta x}{A_{23}},
$$

where $A_{23}=x_{2}^{\prime} y_{3}^{\prime}-x_{3}^{\prime} y_{2}^{\prime}$ is twice the area of the triangle 123, $\Delta x=x_{3}^{\prime}-x_{2}^{\prime}$ and $\Delta y=y_{3}^{\prime}-y_{2}^{\prime}$. Substituting (12), (8) and their derivatives into (11) (considering for example six triangles as in Fig. 3) results in

$$
I_{23}+I_{34}+I_{45}+I_{56}+I_{67}+I_{72}=0,
$$

where, for the first triangle

$$
\begin{aligned}
I_{23}= & \int_{\Omega_{23}}\left[-\frac{\Delta y}{A_{23}} k(T)\left(b+d y^{\prime}+2 e x^{\prime}\right)\right. \\
& +\frac{\Delta x}{A_{23}} k(T)\left(c+d x^{\prime}+2 f y^{\prime}\right) \\
& \left.-\left(1-\frac{\Delta y}{A_{23}} x^{\prime}+\frac{\Delta x}{A_{23}} y^{\prime}\right) \dot{q}(x, y)\right] \mathrm{d} x^{\prime} \mathrm{d} y^{\prime}
\end{aligned}
$$

and similar integrals define the rest of the triangles. The number of integrals for which this process should be carried out is identical to the number of neighboring points we consider.

The calculation of the integral is simple only for the case when $\dot{q}$ is uniform and $\alpha=0$. In this case the discretized PDE obtained is identical to that obtained by the collocation method (that is if we substitute $\alpha=0$ in eqn (9)). As a more general example, consider variable conductivity as defined in (7) and heat source that depends on the $x$ coordinate as

$$
\dot{q}(x)=q_{0}\left(w_{0}+w_{1} x^{2}+w_{2} x^{4}+w_{3} x^{6}\right) .
$$

Note that $x$ is the global coordinate and when substituting (14) into (13), we used $x=x_{1}+x^{\prime}$. The substitution and the integration of (13) was performed by a computer program for symbolic manipulation. The following is the resulting expression for the first triangle

$$
I_{23}=2 e+2 f+C_{\alpha} \alpha+C_{q} \frac{\dot{q}}{K_{s}},
$$

where

$$
\begin{aligned}
C_{q}= & w_{0}+w_{1}\left(10 x_{1}^{2}+5 x_{1} x_{2}^{\prime}+x_{2}^{\prime 2}+5 x_{1} x_{3}^{\prime}\right. \\
& \left.+x_{2}^{\prime} x_{3}^{\prime}+x_{3}^{\prime 2}\right) / 10+w_{2}\left(35 x_{1}^{4}+35 x_{1}^{3} x_{2}^{\prime}\right. \\
& +21 x_{1}^{2} x_{2}^{\prime 2}+7 x_{1} x_{2}^{\prime 3}+x_{2}^{\prime 4}+35 x_{1}^{3} x_{3}^{\prime} \\
& +21 x_{1}^{2} x_{2}^{\prime} x_{3}^{\prime}+7 x_{1} x_{2}^{\prime 2} x_{3}^{\prime}+x_{2}^{\prime 3} x_{3}^{\prime}+21 x_{1}^{2} x_{3}^{\prime 2} \\
& \left.+7 x_{1} x_{2}^{\prime} x_{3}^{\prime 2}+x_{2}^{\prime 2} x_{3}^{\prime 2}+7 x_{1} x_{3}^{\prime 3}+x_{2}^{\prime} x_{3}^{\prime 3}+x_{3}^{\prime 4}\right) / 35 \\
& +w_{3}\left(84 x_{1}^{6}+126 x_{1}^{5} x_{2}^{\prime}+126 x_{1}^{4} x_{2}^{\prime 2}+84 x_{1}^{3} x_{2}^{\prime 3}\right. \\
& +36 x_{1}^{2} x_{2}^{\prime 4}+9 x_{1} x_{2}^{\prime 5}+x_{2}^{\prime 6}+126 x_{1}^{5} x_{3}^{\prime} \\
& +126 x_{1}^{4} x_{2}^{\prime} x_{3}^{\prime}+84 x_{1}^{3} x_{2}^{\prime 2} x_{3}^{\prime}+36 x_{1}^{2} x_{2}^{\prime 3} x_{3}^{\prime} \\
& +9 x_{1} x_{2}^{\prime 4} x_{3}^{\prime}+x_{2}^{\prime 5} x_{3}^{\prime}+126 x_{1}^{4} x_{3}^{\prime 2}+84 x_{1}^{3} x_{2}^{\prime} x_{3}^{\prime 2} \\
& +36 x_{1}^{2} x_{2}^{\prime 2} x_{3}^{\prime 2}+9 x_{1} x_{2}^{\prime 3} x_{3}^{\prime 2}+x_{2}^{\prime 4} x_{3}^{\prime 2}+84 x_{1}^{3} x_{3}^{\prime 3} \\
& +36 x_{1}^{2} x_{2}^{\prime} x_{3}^{\prime 3}+9 x_{1} x_{2}^{\prime 2} x_{3}^{\prime 3}+x_{2}^{\prime 3} x_{3}^{\prime 3}+36 x_{1}^{2} x_{3}^{\prime 4} \\
& \left.+9 x_{1} x_{2}^{\prime} x_{3}^{\prime 4}+x_{2}^{\prime 2} x_{3}^{\prime 4}+9 x_{1} x_{3}^{\prime 5}+x_{2}^{\prime} x_{3}^{\prime 5}+x_{3}^{\prime 6}\right) / 84
\end{aligned}
$$

and

$$
\begin{aligned}
C_{\alpha}= & b^{2}+c^{2}+2 a(e+f)+\frac{c}{2}\left(d\left(x_{2}^{\prime}+x_{3}^{\prime}\right)\right. \\
& \left.+e\left(y_{2}^{\prime}+y_{3}^{\prime}\right)+3 f\left(y_{2}^{\prime}+y_{3}^{\prime}\right)\right) \\
& +\frac{b}{2}\left(f\left(x_{2}^{\prime}+x_{3}^{\prime}\right)+3 e\left(x_{2}^{\prime}+x_{3}^{\prime}\right)\right. \\
& \left.+d\left(y_{2}^{\prime}+y_{3}^{\prime}\right)\right)+\frac{3}{5} e^{2}\left(x_{2}^{\prime 2}+x_{2}^{\prime} x_{3}^{\prime}+x_{3}^{\prime 2}\right) \\
& +\frac{3}{5} f^{2}\left(y_{2}^{\prime 2}+y_{2}^{\prime} y_{3}^{\prime}+y_{3}^{\prime 2}\right)+\left(\frac{d^{2}}{10}+\frac{e f}{5}\right) \\
& \times\left(x_{2}^{\prime 2}+x_{2}^{\prime} x_{3}^{\prime}+x_{3}^{\prime 2}+y_{2}^{\prime 2}+y_{2}^{\prime} y_{3}^{\prime}+y_{3}^{\prime 2}\right) \\
& +\frac{3}{10} d\left((e+f)\left(2 x_{2}^{\prime} y_{2}^{\prime}+x_{3}^{\prime} y_{2}^{\prime}+x_{2}^{\prime} y_{3}^{\prime}+2 x_{3}^{\prime} y_{3}^{\prime}\right)\right) .
\end{aligned}
$$

This was implemented in a Fortran computer program. In eqn (15) terms were discarded. These are the terms that cancel each other when the summation of (13) is performed. Although the result $(13,15)$ is more complex than (9), it is similar in principle.

Equations (9) or (13), to which we will refer hereafter as the discretized PDE, are the only ones in 
this algorithm, that are unique to the present example of heat conduction. When implementing this algorithm to a computer code, the relation of the discretized PDE takes place as a separate subroutine that can be easily replaced whenever a different PDE is to be solved. From now on, the formulation of the algorithm is general and is applicable to any boundary value problem.

\subsection{The compatibility requirements}

The above-mentioned discretized PDE provides one out of at least six conditions, needed at each point, in order to determine the six unknown coefficients of its jet. The other conditions are produced by the compatibility requirements. We require that at any neighboring node $j$, the value of the jet about the node $i$ (the origin) and the value of the jet about node $j$, be compatible. If $j$ is an internal node then the condition is

$$
a_{i}+b_{i} h_{x}+c_{i} h_{y}+d_{i} h_{x} h_{y}+e_{i} h_{x}^{2}+f_{i} h_{y}^{2}-a_{j}=0 \pm \epsilon
$$

If $j$ is on the boundary, either

$$
a_{i}+b_{i} h_{x}+c_{i} h_{y}+d_{i} h_{x} h_{y}+e_{i} h_{x}^{2}+f_{i} h_{y}^{2}=T_{j} \pm \epsilon
$$

or

$$
\begin{aligned}
& b_{i} \cos \gamma+c_{i} \sin \gamma+d_{i}\left(h_{x} \sin \gamma+h_{y} \cos \gamma\right) \\
& \quad+e_{i} 2 h_{x} \cos \gamma+f_{i} 2 h_{y} \sin \gamma=-\frac{\dot{q}_{j}}{k\left(T_{j}\right)} \pm \epsilon^{\prime}
\end{aligned}
$$

apply. In (16-18) the permissible errors are

$$
\epsilon=\epsilon_{0}|h|^{3}, \quad \epsilon^{\prime}=\epsilon_{0} h^{2},
$$

and $h_{x}$ and $h_{y}$ are the $x$ and $y$ components (respectively) of the relative position vector, $h$, between $i$ and $j$ (see Fig. 2). Equation (17), states that the expansion of $T_{i}$ at a neighboring node $j$ on the boundary, should approximate the prescribed temperature $T_{j}$. For neighboring nodes on a boundary with prescribed flux, eqn (18) is used. It is the result of using the components of discretized temperature gradients $\partial T / \partial x\left(x_{j}^{\prime}, y_{j}^{\prime}\right)$ and $\partial T / \partial y\left(x_{j}^{\prime}, y_{j}^{\prime}\right)$ to calculate the normal heat flux and comparing it to the boundary condition. The error permitted in the flux boundary condition is computed with an order of expansion which is one lower than the former continuity conditions, since the Taylor expansion was differentiated once.

The positive real number $\epsilon_{0}$ in eqns (19), is a constant used to evaluate the order of the permissible

† In the following equations the following matrix notation is used: $[\cdot]$ denotes a matrix, $\{\cdot\}$ a column vector and $L \cdot]$ a row vector. discrepancy according to the relation $\epsilon=O\left(h^{k+1}\right)$ for the truncation error (in our case the polynomial order, $k$, is equal to two). One may take $\epsilon_{0}$ as one initially. After an approximate solution is computed, the error may be evaluated, and a better estimation for $\epsilon_{0}$ can be obtained.

In order to obtain the minimal number of conditions so that the coefficients of the jets can be determined uniquely, compatibility equations with five neighboring nodes should be written as in [1]. Then, having six equations for each internal point, and $N$ arbitrarily distributed points in the domain, one may write a system of $6 \mathrm{~N}$ equations and solve it for the $6 \mathrm{~N}$ unknown coefficients of the various jets. A morc general approach would consider a variable number of $n>5$ neighboring points that would yield an overdetermined set of $(n+1) N$ equations. As discussed in the introduction, this has the advantages of better geometrical flexibility and improved accuracy $[4,5]$. Henceforth it is assumed that $n>5$ is the number of compatibility equations used for the jet corresponding to any node $i, i=1, \ldots, N$.

If possible, an effective way to solve the problem would be to treat first the blocks of $n+1$ equations corresponding to each node ( $n$ compatibility equations and one discretized PDE), so that each such block is reduced to one equation. In such a way, we will have to solve eventually a system of $N$ equations rather than a system of at least $6 N$ equations (in case we take the minimal $n=5$ compatibility equations). It turns out that it is convenient to reduce the block of $(n+1)$ equations obtained for the node $i$ to a single equation in the coefficient $a_{i}$ and the $n$ coefficients $a_{j}$ for the neighboring nodes. The procedure for the reduction of such a block also takes care of the fact that the set of $(n+1) N$ equations is overdetermined.

The block of $n+1$ equations may be written in matrix form as $\dagger$

$$
[K]\{A\}=\{F\} \pm\{E\}
$$

where $[K]$ is of size $(n+1) \times(n+6),\{A\}$ is a column vector of dimension $n+6$ which contains the six unknown polynomial coefficients of point $i$ in addition to $n$ coefficients, the $a$ coefficients of the neighboring points. $\{F\}$ and $\{E\}$ are column vectors of dimension $n+1$ containing the right-hand sides of conditions (9) or (13) and (16) to (18). Equation (20) is then segmented, dividing $\{A\}$ to $\left\{A_{1}\right\}=\left\{a_{i}, a_{j_{1}}, \ldots, a_{j_{n}}\right\}$ and $\left\{A_{2}\right\}=\left\{b_{i}, c_{i}, d_{i}, e_{i}, f_{i}\right\}$, and so (20) breaks up into two equations

$$
\left\lfloor K_{11}\right\rfloor\left\{A_{1}\right\}+\left\lfloor K_{12}\right\rfloor\left\{A_{2}\right\}=F_{1}
$$

which is the vector form of the discretized PDE (9) or (13), and

$$
\left[K_{21}\right]\left\{A_{1}\right\}+\left[K_{22}\right]\left\{A_{2}\right\}=\left\{F_{2}\right\} \pm\left\{E_{2}\right\}
$$


which is the matrix form of the $n$ compatibility or boundary conditions from (16)-(18). The set of $n+1$ equations with $n+6$ unknowns (20), can be replaced by the following single equation with $n+1$ unknowns

$$
\begin{aligned}
\left\lfloor\lfloor Q\rfloor\left[K_{21}\right]-\left\lfloor K_{11}\right\rfloor\left\{A_{1}\right\}=\right. & \lfloor Q\rfloor\left\{F_{2}\right\} \\
& -F_{1} \pm\lfloor Q\rfloor\left\{E_{2}\right\},
\end{aligned}
$$

where

$$
\lfloor Q\rfloor=\left\lfloor K_{12}\right\rfloor\left[\left[K_{22}\right]^{T}\left[K_{22}\right]\right]^{-1}\left[K_{22}\right]^{T} .
$$

In order to obtain (23) from (21), (22), some matrix manipulations were performed. In particular, we used a scheme for solving an overdetermined set of linear equations of the form $[K]\{x\}=\{y\}$. In such a case, one premultiplies both sides of the set by the transpose of the matrix: $[K]^{T}[K]\{x\}=[K]^{T}\{y\}$ to obtain the normal equations of the least square problem [9].

\subsection{The solution of the equations}

The single row (23), expresses the relation between the coefficients $a_{i}, a_{j_{1}}, \ldots, a_{i_{n}}$ for each nodal point. It is substituted in a global matrix in the form of eqn (20), which relates the $a$ coefficients of all the points inside the domain. From the definition of the jet (8) it is clear that the value of the unknown dependent variable at any point $i$ is $a_{i}$. Hence, if in a linear case, only the values of $T$ at the nodal points are needed, the solution of global matrix equation provides the complete solution of the problem.

It is only natural that the system of equations we obtained in the NTDM will be solved by an iterative method so that the accuracy of their solution will be according to the truncation error we expect. With this in mind, the solution is done in the present work by the Gauss-Seidel iterative method with successive over-relaxation [10]. The conditions of permissible discrepancy are used when the associated error $E_{i}$ for each local relation (23) becomes the criterion for convergence of the dependent variable $a_{i}$ when solving the global matrix by iterations. Stopping the iterative process whenever the values of the respective dependent variables are changed by magnitude that is less than $E_{i}$, enables us to control the magnitude of the discrepancy according to an estimation of the truncation error that would result anyway from the order of jets and grid spacing. The ability to save computation time using this method depends on whether it is possible to use an iterative method, that is if the solution will converge. Accelerating and assuring convergence may be done using some other iterative methods [10] which are getting more attention during the last decade [11] and are being used also by the FEM.

In a nonlinear problem, or in the case when values of $T$ between nodes are also needed, it is necessary to obtain the rest of the polynomial coefficients, $b_{i} \cdots f_{i}$.
This is done by rewriting the relations (16)-(18), this time the coefficients $a_{i}$ are known. Thus, for each node, an $n \times 5$ overdetermined matrix is obtained and solved separately by the normal equations method. A nonlinear problem is solved by first assuming values for $b_{i} \cdots f_{i}$, and after a linear solution is obtained, these coefficients are determined and are substituted into the right-hand side of the discretized PDE (9) or (13). Then, the whole process is performed iteratively until the successive values of coefficients $a_{i}$ differ in less than $E_{i}$. Hence, the nonlinear iterative procedure, as well as the whole solution, are performed until all local desired accuracies are obtained.

The procedure described above is very efficient for nonlinear problems, since the number of Gauss-Seidel iterations needed for the solution of the global matrix, decreases with nonlinear iteration steps. This is achieved by using the latest nonlinear iteration's solution as an initial guess for the next Gauss-Seidel solution of the global matrix.

\section{A ONE-DIMENSIONAL TEST CASE}

A one-dimensional test case of nonlinear conduction, is considered, (eqn (6) is taken for the $x$ direction only). The conductivity is temperature dependent (7) and heat generation may be nonuniform (14). The boundary conditions for the problem are

$$
\left.\frac{\partial T}{\partial x}\right|_{x=0}=0 ;\left.\quad T\right|_{x=L}=T_{L} .
$$

The exact solution for this problem is

$$
T(x)=\frac{-1+\sqrt{2 \alpha F(x)+\left(1+\alpha T_{L}\right)^{2}}}{\alpha},
$$

where

$$
\begin{aligned}
F(x)=\frac{q_{0}}{2 k_{s}}[ & w_{0}\left(L^{2}-x^{2}\right)+\frac{w_{1}}{6}\left(L^{4}-x^{4}\right) \\
& \left.+\frac{w_{2}}{15}\left(L^{6}-x^{6}\right)+\frac{w_{3}}{28}\left(L^{8}-x^{8}\right)\right] .
\end{aligned}
$$

The numerical solution by the NTDM is performed using different modes, namely with five or eight neighbors, in the collocations (differential) or weighted residuals (integral) formulations, and in combinations of those, in order to verify the algorithm in those modes. The numerical computation was performed using a gencral purpose two-dimensional code, but since the problem is one-dimensional the results in the $y$ direction and the number of grid points in that direction are irrelevant. Figure 4 shows the absolute relative errors obtained in each case as a function of the number of grid point in the $x$ direction. The relative error is defined as the mean of ratios of the absolute error to the maximum of the 


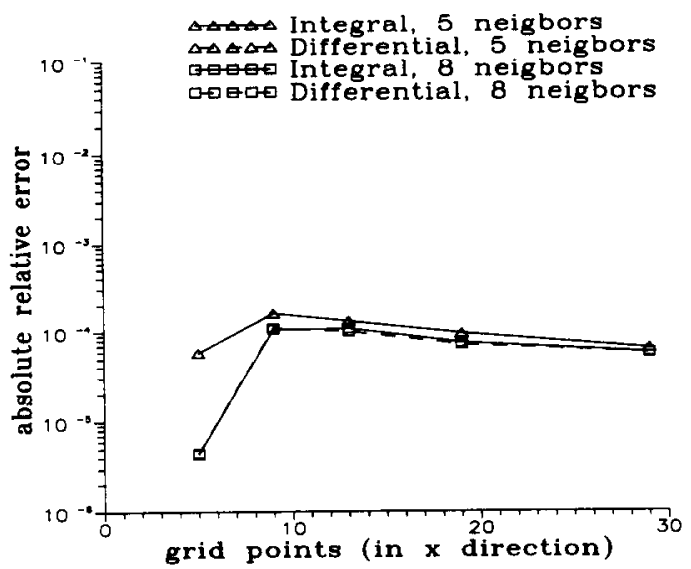

Fig. 4(a).

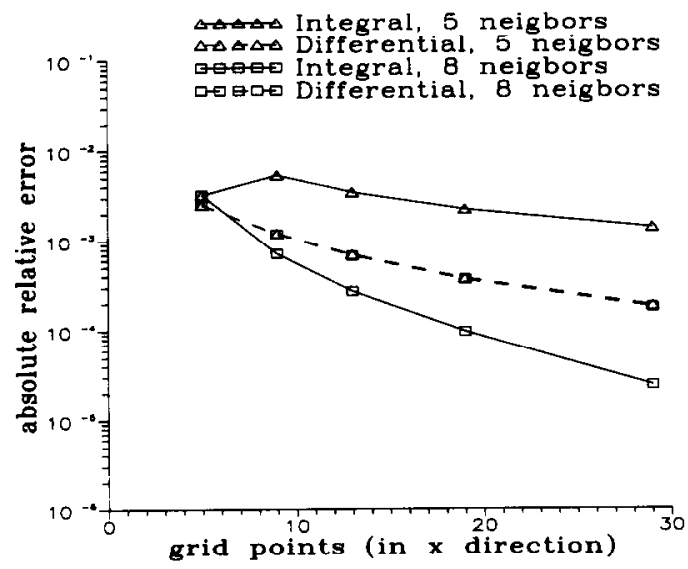

Fig. 4(c).

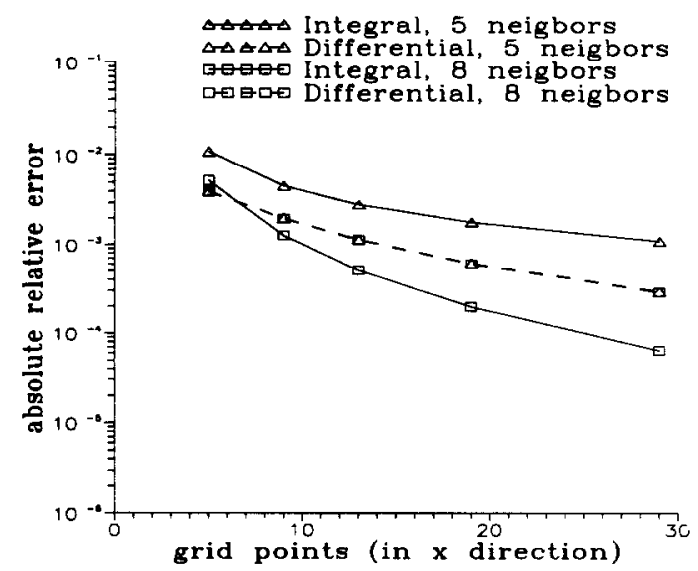

Fig. 4(b).

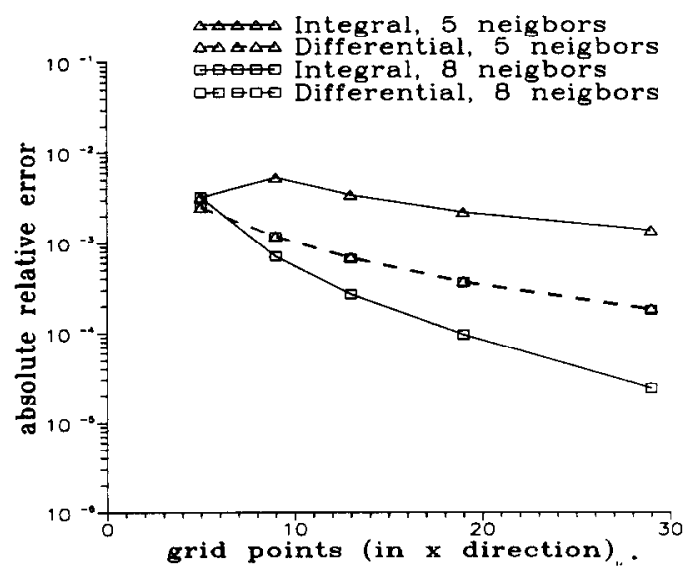

Fig. 4(d).

Fig. 4. Relative error vs number of grid points in various cases. (Linear- $\alpha=0, k_{s}=0.2738$, nonlinear$\alpha=8.439 \times 10^{-4}, k_{s}=0.3294$, uniform $-w_{i}=\{1,0,0,0\}$, nonuniform $-w_{i}=\{1,-1,-0.5,0.1\}$.)

temperature distribution (obtained by the exact solution for each case). This was done in order to normalize the error, as the temperature distributions vary from case to case. Each case (a)-(d) is the result of applying the NTDM to a different problem, namely either to uniform or nonuniform heat generation $\dot{q}$, and to either a linear or nonlinear problem (temperature independent or dependent conductivity, respectively).

It is clear from all the graphs in Fig. 4 that the integral formulation (continuous curves) is more accurate and converges faster than the collocations formulation (dashed curves). In the linear and uniform case (a), accuracy is good in all models even for coarse grids. It seems that there is no advantage in increasing the number of neighboring points with the collocations formulation, but this may be different in a more general test case. It was observed that the errors in the calculations are either above the exact solution or below it depending on the method of implementation of the NTDM used. This phenomenon cannot be observed in the graphs since they show absolute values of the errors only. When considering the signs of the errors, it seems that when integral formulation results are upperbounds, the collocations formulation results are lower bounds, and vice-versa. The phenomenon may be useful in order to verify that convergence is achieved by comparing results of both formulations. It is particularly meaningful in nonlinear problems where it is difficult to determine when convergence is reached.

\section{TWO-DIMENSIONAL TEST CASES}

The purpose of the following two-dimensional test cases of nonlinear conduction is to demonstrate the ability of the NTDM to handle complex geometries and different boundary conditions, and to compare the results to those obtained by the FEM.

Firstly, we consider a two-dimensional region with uniformly distributed internal heat source and ternperature dependent conductivity. Figure 5 shows the mesh for the region as done by an automatic mesh generator for the FEM. Two mesh densities are shown, a basic coarse mesh (Fig. 5a) and a finer mesh (Fig. 5b) obtained by dividing each element of the coarse mesh to four elements. Note the irregularity of the meshes. A pre-processor that reads the data of the 


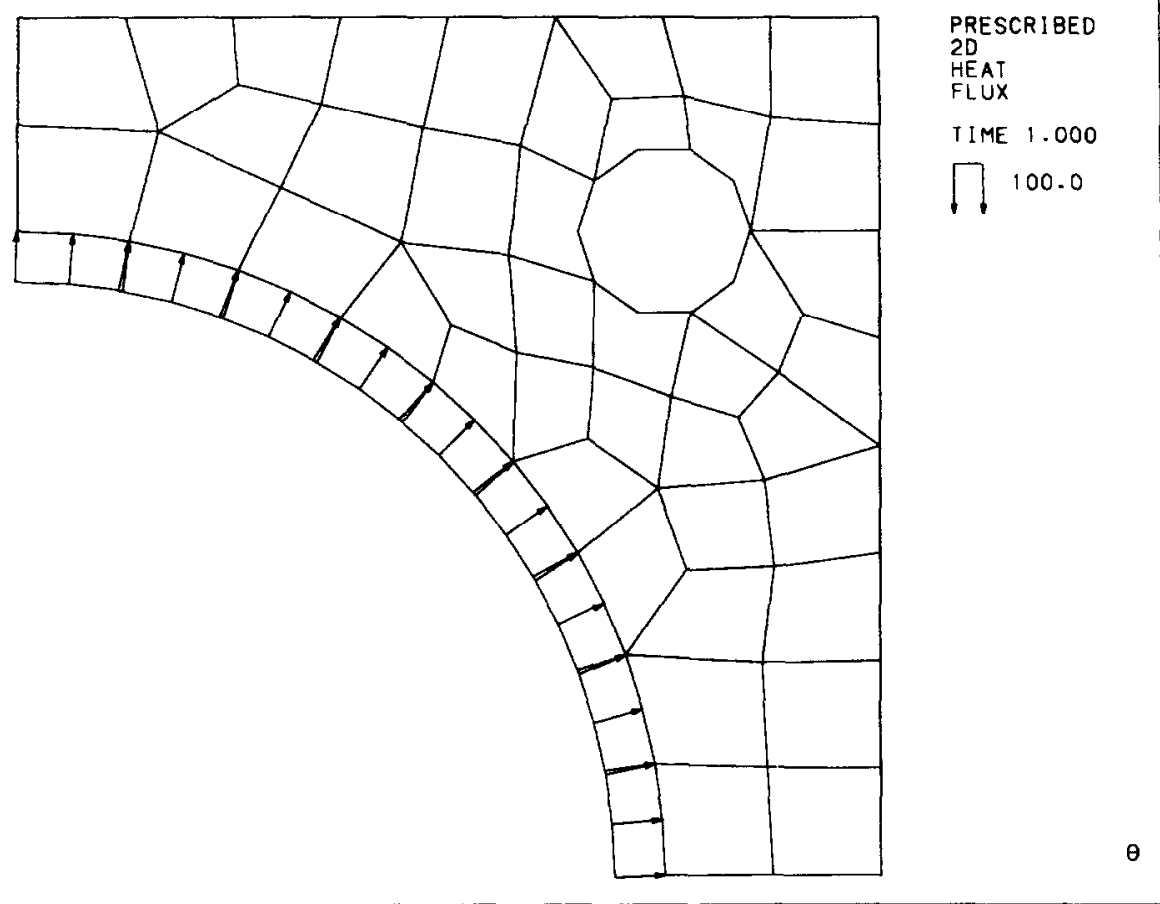

Fig. 5(a). FEM course mesh.
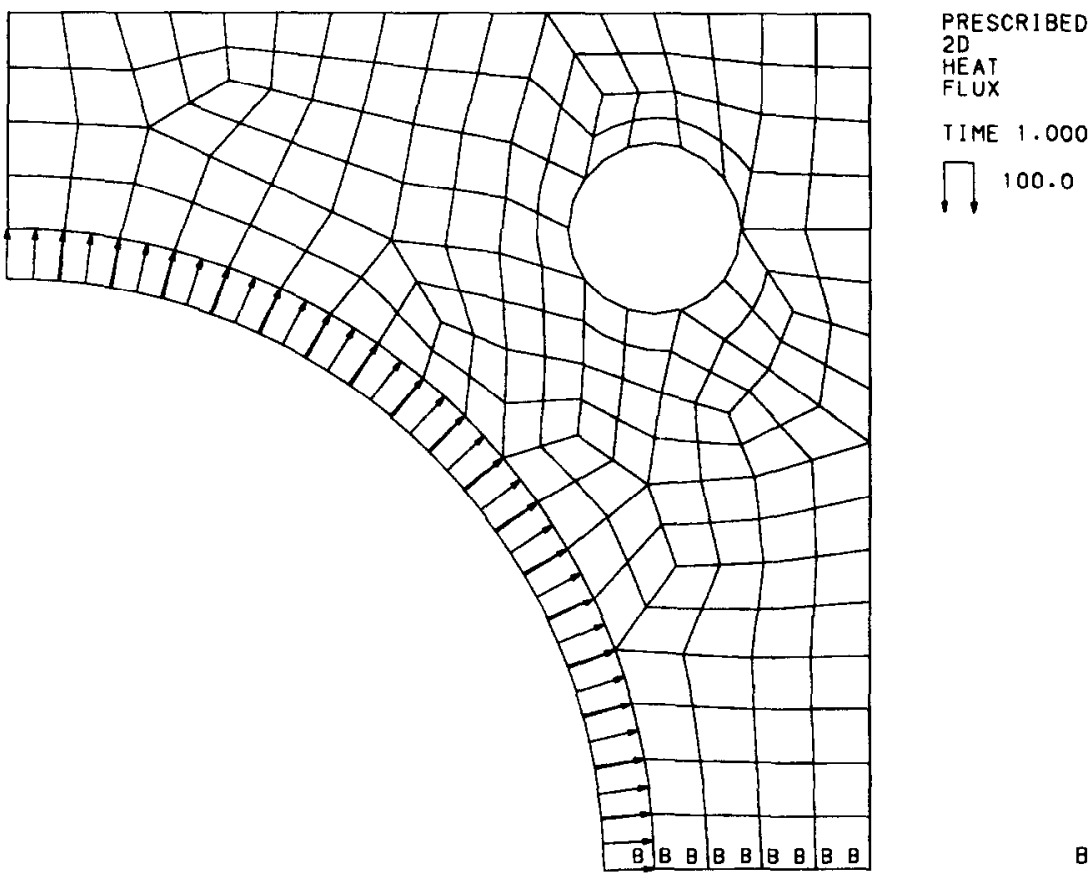

Fig. 5(b). Four-fold refined FEM mesh.

FEM mesh and generates automatically the input for the NTDM code was written. A plot of this mesh is of no interest since the NTDM grid uses the same nodes as the FEM.
On these meshes two cases of boundary conditions were considered. In the first case we specify heat flux through the arc (as shown in Fig. 5), constant temperatures on the upper and lower boundaries, and the 


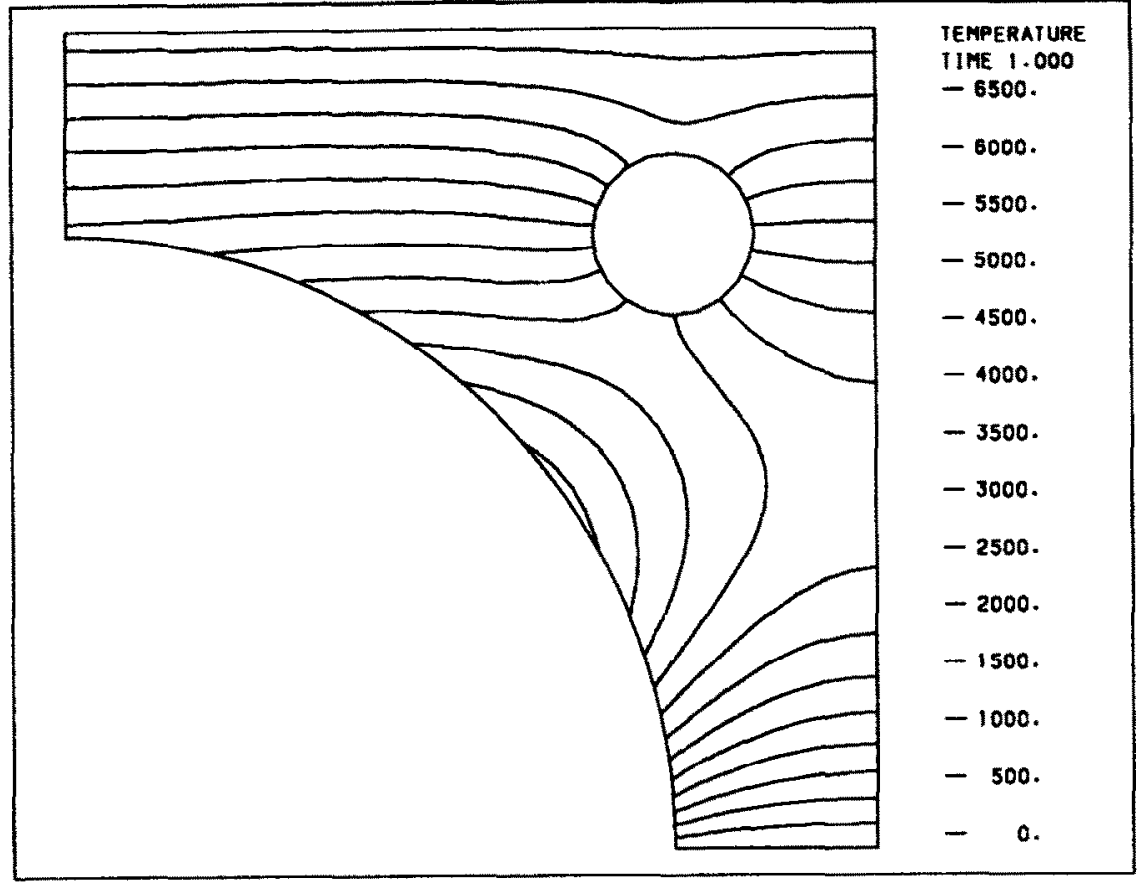

Fig. 6(a). FEM result for case 1.

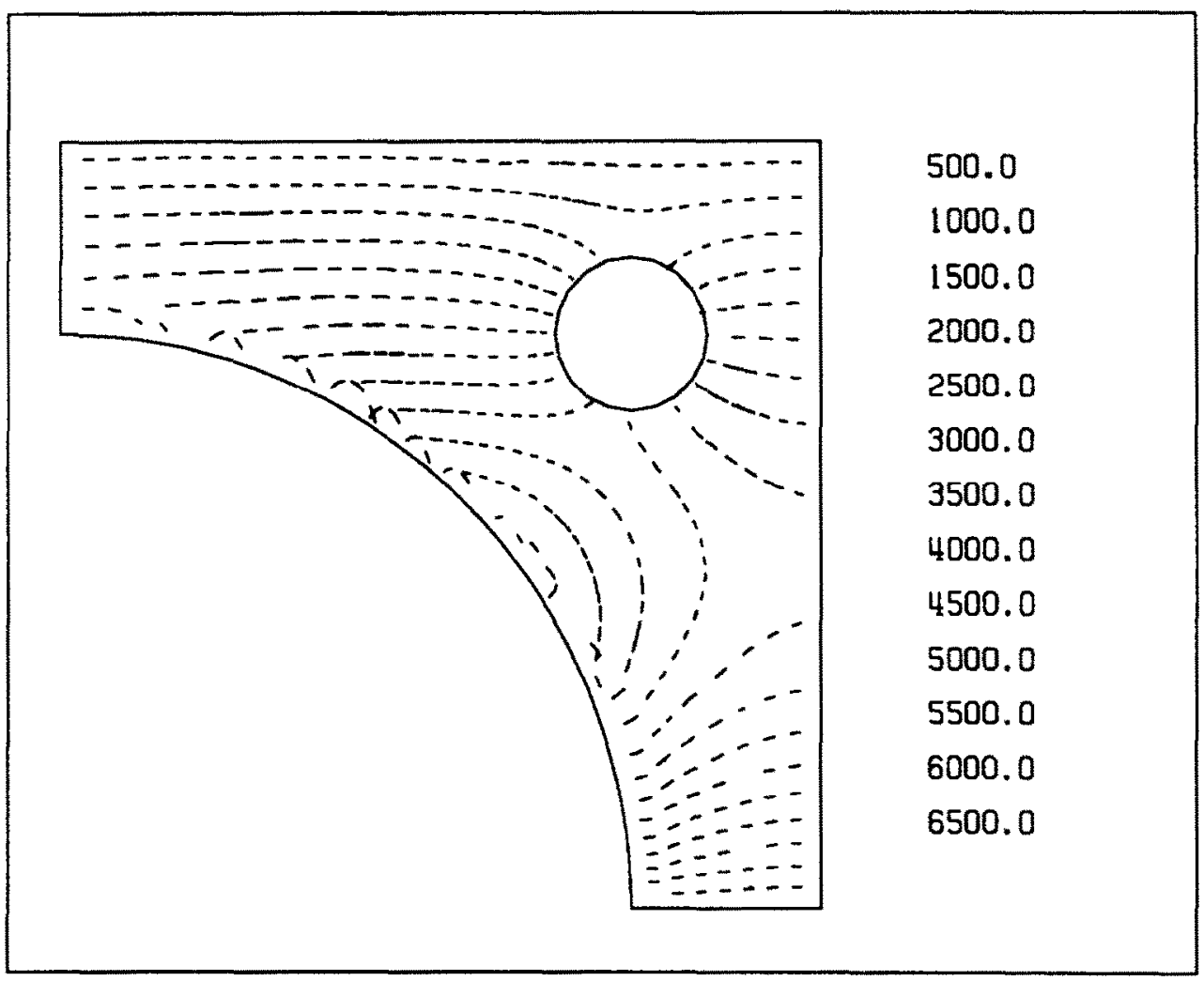

Fig. 6(b). NTDM result for case 1 .

left, right and hole boundaries are insulated. Figure 6 shows the result of the computations as obtained by FEM (Fig. 6a) and by the NTDM (Fig. 6b) for the finer mesh (Fig. 5b). The FEM program used here has a post-processor which produces quality drawings of constant temperature contours (Fig. 6a). For the task of drawing the contours of constant temperature it is possible to develop an algorithm that takes advantage 


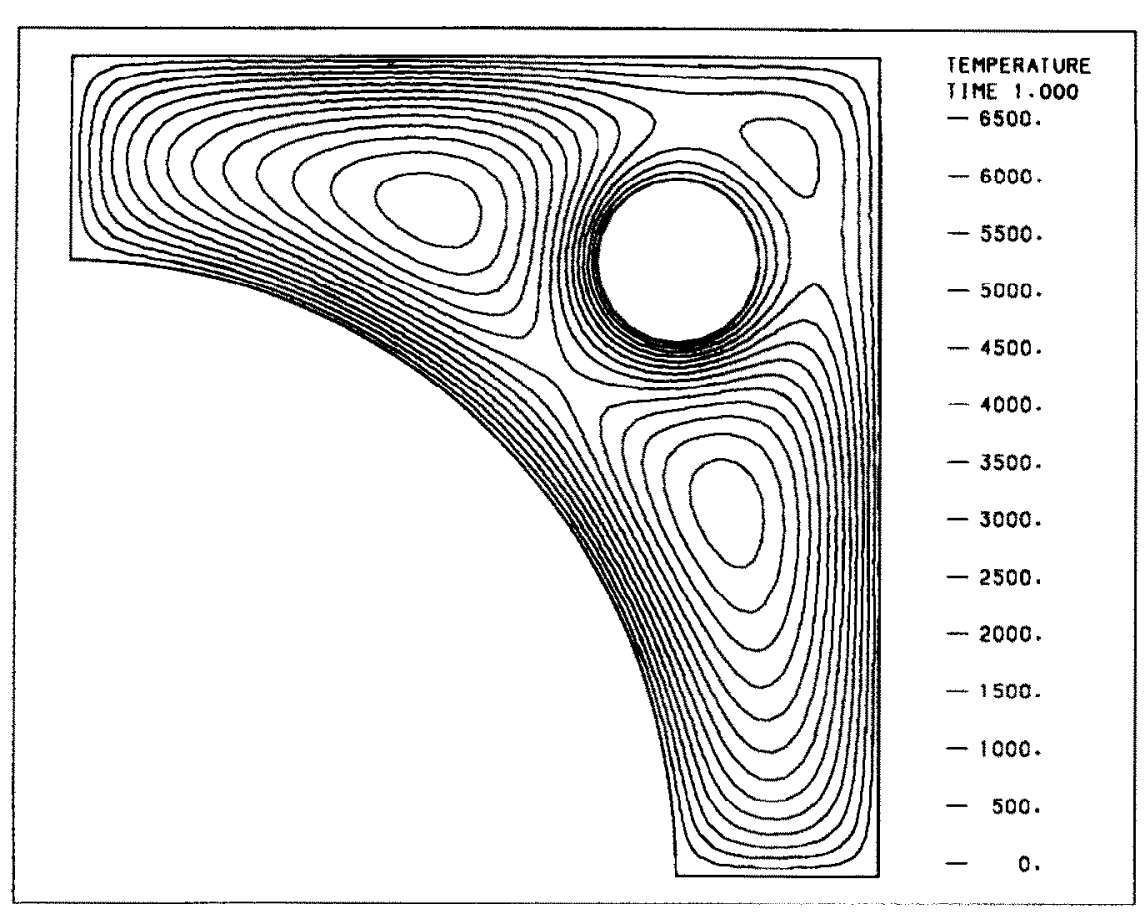

Fig. 7(a), FEM result for case 2.

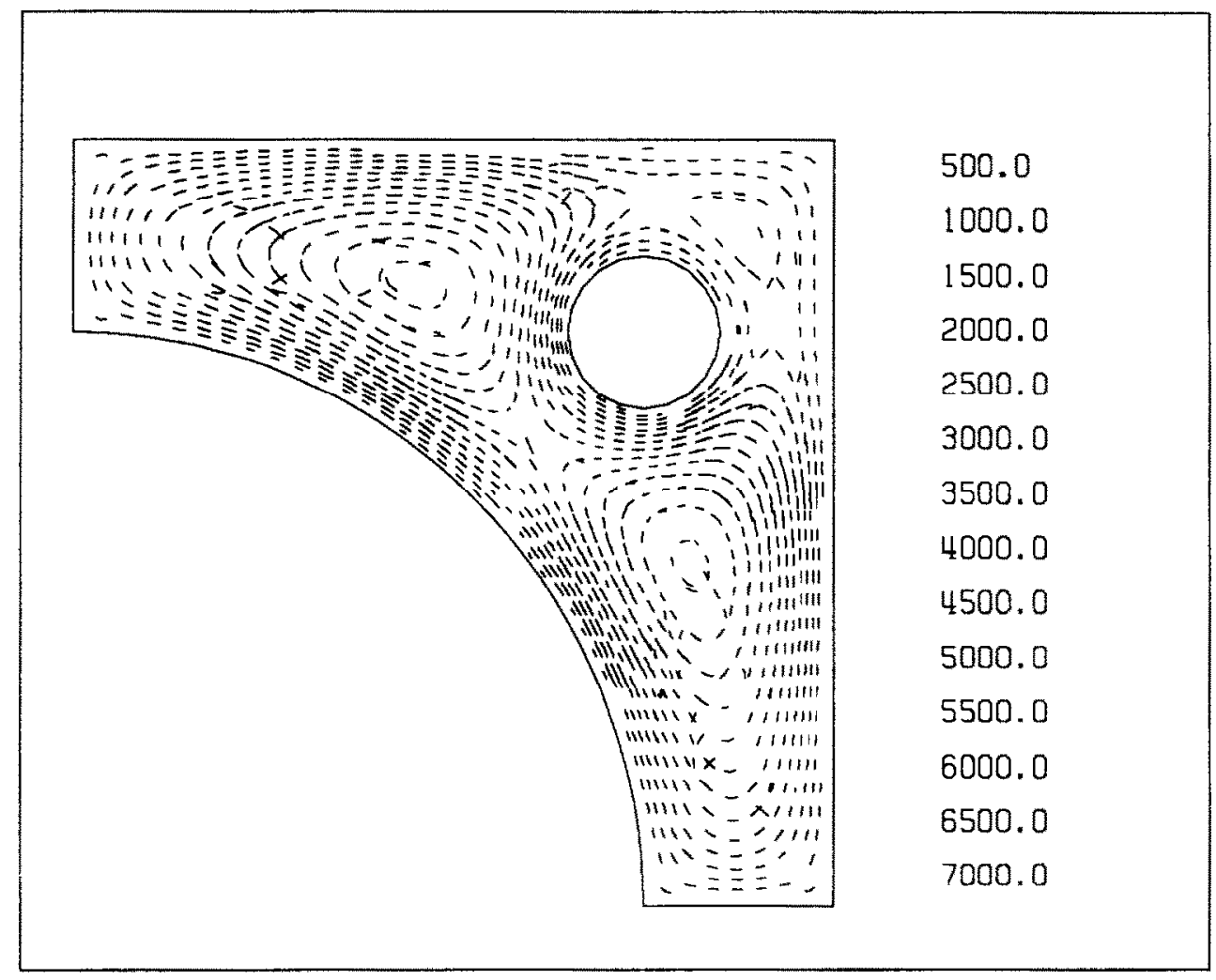

Fig. 7(b). NTDM for case 2.

of the fact that the results obtained in the NTDM are in the form of jets about the various nodes. We used a primative program that follows the jet approach and produces less accurate but faster drawings
(Fig. 6b). The qualitative comparison of the figures shows good agreement between the methods.

For the second case we consider the same region and material where all the boundaries are at $T=0$. 


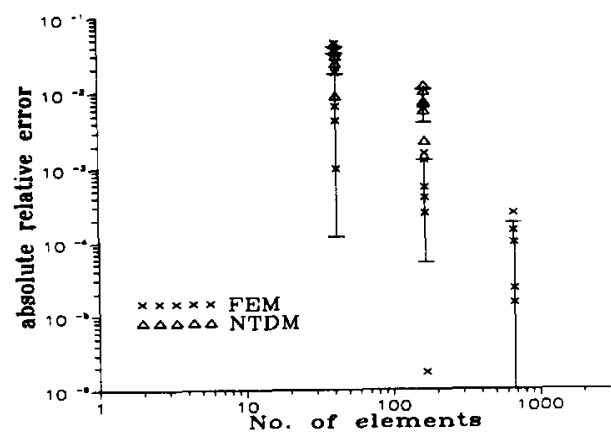

Fig. 8. Comparison of absolute errors of FEM and NTDM results.

The results for this test case using the NTDM and the FEM are shown in Fig. 7. Good agreement between the methods is manifested also in this case.

To compare the errors obtained, an additional study of the second test case was performed. Using the FEM, the mesh of Fig. 5(b) was refined in two more stages, thus providing results of the same problem for four degrees of refinement of the same region. Since an analytical result is not available for this problem, and since the FEM results seemed to converge with mesh refinement, we considered the finest mesh's results as the 'accurate result'.

Resulting temperatures for six selected nodes were compared to this accurate result, and the absolute relative error was plotted in Fig. 8 for both methods as a function of grid density (number of elements). The NTDM computation was performed only for the two coarsest grids. The FEM results of the finest grid are not plotted for the obvious reason that they serve as a reference. The results show that both the FEM and NTDM converge, although the NTDM does not converge as fast or may converge to a slightly different solution. The differences may be small from an engineering point of view, but the ability to solve the same problem by different methods with a little additional effort may be of advantage when considering nonlinear problems. This may increase one's trust in the numerical results.

\section{DISCUSSION}

In [1] the following advantages of the nonconforming Taylor discretization method were considered: the convenient implementation of various partial differential equations, the efficient solution of the algebraic equations and the geometrical flexibility that the method allows.
Here, we suggest improved application of the general scheme using a variable number of neighboring nodes for the compatibility conditions and weighted residual discretization of the PDE.

The use of the method of weighted residuals is more complicated than the collocation method used in [1]. However, using a symbolic manipulator the discretized PDE is readily obtained. The resulting improved accuracy is evident in the cases considered.

The use of the variable number of neighboring nodes further improves the geometrical flexibility. In addition, as noted in [4], a singular system of equations may be obtained if neighboring nodes are not chosen properly. An overdetermined set of continuity conditions will result a non-singular set of equations. The possibility of having a variable number of points and the method specified for the manipulation of the compatibility equations will prevent the singularity. This is of importance particularly if the neighboring nodes for the compatibility conditions are chosen by computer automatically from an arbitrary grid.

\section{REFERENCES}

1. E. Kochavi, R. Segev and Y. Yomdin, Numerical solution of field problems by nonconforming Taylor discretization. Appl. Math. Modelling 15, 152-157 (1991).

2. Y. Yomdin and E. Elihai, Flexible high order discretization. Preprint, Weizmann Institute of Science (1989).

3. A. N. Kolmogorov and V. M. Tihomirov, $\epsilon$-Entropy and $\epsilon$-capacity of sets in functional spaces. AMS Transl. 2, 17 (1961).

4. N. Perrone and R. Kao, A general finite difference method for arbitrary meshes. Comput. Struct. 5, 45-58 (1975).

5. T. Liszka and J. Orkisz, The finite difference method at arbitrary irregular grids and its application in applied mechanics. Comput. Struct. 11, 83-95 (1980).

6. M. Arad, Application of the nonconforming Taylor discretization method to two dimensional elasticity. M.Sc. thesis, Ben-Gurion University of the Negev (in Hebrew) (1992).

7. P. J. M. Sonnemans, L. P. H. De Goey and J. K. Nieuwenhuizen, Optimal use of a numerical method for solving differential equations based on Taylor series expansions. Int. J. Numer. Meth. Engng 32, 471-499 (1991).

8. Y. Yomdin and Z. Wiener, Jet solving of ODE. Preprint, Weizmann Institute of Science (1992).

9. W. H. Press et al., Numerical Recipes. Cambridge University Press, Cambridgc (1989).

10. D. M. Young, Iterative Solution of Large Linear Systems. Academic Press, New York (1971).

11. F. Angeleri, V. Sonnad and K.-J. Bathe. Studies of finite element procedures - an evaluation of preconditioned iterative solvers. Comput. Struct. 32, 671-677 (1989). 